\title{
Outcome of Off-Label AREDS 2 Supplementation for the Treatment of Macular Degeneration in Non-Proliferative Idiopathic Type 2 Macular Telangiectasia
}

This article was published in the following Dove Press journal:

Clinical Ophthalmology

\author{
Tyler A Berger' \\ Matthew W Manry ${ }^{1,2}$ \\ Lucas B Lindsell ${ }^{1,2}$ \\ James M Osher ${ }^{1,2}$ \\ Daniel M Miller ${ }^{1,2}$ \\ Robert E Foster ${ }^{1,2}$ \\ Christopher D Riemann ${ }^{1,2}$ \\ Michael R Petersen ${ }^{1,2}$ \\ Robert A Sisk ${ }^{1-3}$ \\ 'Department of Ophthalmology, \\ University of Cincinnati, Cincinnati, $\mathrm{OH}$, \\ USA; ${ }^{2}$ Cincinnati Eye Institute, Cincinnati, \\ $\mathrm{OH}$, USA; ${ }^{3}$ Abrahamson Pediatric Eye \\ Institute, Cincinnati Children's Hospital \\ Medical Center, Cincinnati, OH, USA
}

Purpose: To evaluate if off-label Age-Related Eye Disease Study 2 (AREDS2) supplementation prevents visual and anatomical deterioration in non-proliferative Idiopathic Macular Telangiectasia Type 2 (IMT2).

Patients and Methods: This is a single-center retrospective, comparative study of 82 IMT2 eyes treated with AREDS2 from January 1st, 2013 to January 1st, 2018. The study analysis consisted of a non-comparative arm, which included all AREDS2 eyes, and a comparative arm (27 AREDS2 and 42 untreated eyes) that only included eyes with complete follow-up data. Eyes were evaluated at baseline, 12 and 24 months. Better/worse eye subanalysis was performed in the comparative study arm. Primary outcomes were best corrected visual acuity (BCVA) and optical coherence tomography (OCT) anatomical characteristics including largest cavitation diameter, central macular thickness (CMT), and length of ellipsoid zone (EZ) loss at 24 months.

Results: In the non-comparative arm, AREDS2 eyes showed stable BCVA $(0.28 \pm 0.18$ $\log$ MAR at baseline vs $0.26 \pm 0.19 \log$ MAR at 24 months; $\mathrm{p}=0.35)$ and OCT anatomical features after 24 months of supplementation. In the comparative arm, BCVA mean difference was greater for untreated eyes at 24 months $(-0.09 \pm 0.15$ vs $0.03 \pm 0.11 \operatorname{logMAR} ; \mathrm{p}=$ $<0.001)$. AREDS2 eyes had decreased cavitary diameter and EZ loss compared to untreated eyes at the study endpoint ( $p=0.01$ and $p=0.02$, respectively). CMT remained stable for both cohorts throughout the study. For better/worse eye analysis, untreated eyes had worse BCVA at 24 months in both better and worse eyes (both $\mathrm{p}=0.01$ ). For anatomical outcomes, increases in both EZ loss $(p=0.04)$ and cavitary diameter $(p=0.001)$ among untreated eyes were only significant for eyes with worse baseline BCVA.

Conclusion: Our results suggest that off-label AREDS2 supplementation in non-proliferative IMT2 may prevent anatomical and visual deterioration in a subset of eyes.

Keywords: antioxidant, AREDS2, carotenoid supplementation, ellipsoid zone loss, lutein, idiopathic macular telangiectasia, macular telangiectasia, juxtafoveal macular telangiectasia, parafoveal telangiectasia, retinal vascular disease

\section{Introduction}

Idiopathic Macular Telangiectasia Type 2 (IMT2) is a neurodegenerative disease that primarily affects the central retina. The non-proliferative stage of IMT2 has undergone multiple investigations for effective treatment, including analysis of intravitreal anti-vascular endothelial growth factor injections, argon laser
Correspondence: Robert A Sisk Cincinnati Eye Institute, 1945 CEl Drive, Cincinnati, OH, 45242, USA Fax + I 513-569-3904

Email rsisk@crphealth.com 
photocoagulation, photodynamic therapy, intravitreal triamcinolone, and pars plana vitrectomy with limited evidence of benefit compared to natural history. ${ }^{1-5}$ Encapsulated cell technology releasing ciliary neurotrophic factor is currently being investigated in a Phase III randomized controlled clinical trial (NCT03319849; NCT03316300). ${ }^{6,7}$

Treatment of non-proliferative IMT2 has remained challenging due to the elusiveness of the underlying pathogenesis. While long thought to be predominately a retinal vascular disease, ${ }^{8}$ new insights into the disease process suggest IMT2 may primarily be a neurodegenerative disease with an additional vascular component. ${ }^{9}$ Müller cell loss or dysfunction appears to play a role in the underlying pathophysiology as a result of altered serine metabolism. ${ }^{10}$ A prominent finding in IMT2 is loss of macular luteal pigment. $^{11}$ Histopathological studies have demonstrated that areas of macular pigment depletion correlate with Müller cell loss. ${ }^{12,13}$ The significance of reduced macular pigment density remains uncertain and it is unknown if macular pigment contributes to IMT2 pathophysiology or is a secondary feature. ${ }^{8}$

In the human retina, macular pigment consist of the carotenoids lutein, meso-zeaxanthin and zeaxanthin. ${ }^{9}$ The therapeutic role of carotenoid supplementation in IMT2 has been examined by several small studies. ${ }^{14-16}$ Zeimer et al ${ }^{14}$ and Choi et $\mathrm{al}^{15}$ found increased macular pigment optical density in response to oral carotenoid supplementation but interestingly only in areas where macular pigment was present prior to treatment initiation. Visual acuity outcomes have been variable in response to carotenoid supplementation. A cross-over study by Tan et $\mathrm{al}^{16}$ found visual acuity stabilization after lutein, meso-zeaxanthin and zeaxanthin supplementation. However, a subsequent study by Choi and associates found no beneficial effect of zeaxanthin on visual acuity after 24 months of supplementation.

The purpose of this study is to evaluate if off-label use of a commonly available formulation of carotenoid and antioxidant supplementation in Age-Related Eye Disease Study 2 (AREDS2) may prevent anatomic or visual deterioration in IMT2. We hypothesize that carotenoid and antioxidant supplementation may slow progression of the neurodegenerative disease process and its associated visual decline.

\section{Patients and Methods}

This is a retrospective, comparative chart review of nonproliferative IMT2 treated with off-label AREDS2 at the
Cincinnati Eye Institute in Cincinnati, Ohio. The study protocol was approved by the institutional review board at Cincinnati Children's Hospital Medical Center. We obtained a waiver of The Health Insurance Portability and Accountability Act (HIPPA) authorization for patient informed consent as this study could not be practically conducted otherwise and is of no more than minimal risk. Nonetheless, we did discuss the risks, benefits, and alternatives to off-label AREDS2 prior to initiation of therapy. During this study, patient data remained confidential in accordance with HIPPA regulations and the tenets of the Declaration of Helsinki.

\section{Study Design}

We retrospectively reviewed medical records with a diagnosis of IMT2 (ICD10 codes H35.071, H35.072, and H35.073) from January 1st, 2013 to January 1st, 2018 at the Cincinnati Eye Institute (CEI). Diagnosis of IMT2 was based on clinical exam, spectral-domain optical coherence tomography (SD-OCT), fundus autofluorescence, and fluorescein angiography meeting the criteria described by Yannuzzi et al. ${ }^{17}$ Three retina specialists (RAS, JMO, LBL) at CEI routinely prescribed AREDS2 formula (500mg vitamin C, 400 international units (IU) vitamin E, $80 \mathrm{mg}$ zinc, $2 \mathrm{mg}$ copper, $10 \mathrm{mg}$ lutein and $2 \mathrm{mg}$ zeaxanthin) for patients with non-proliferative IMT2 based upon preliminary evidence of carotenoid supplementation for IMT2, ${ }^{14-16}$ while other providers (REF, DMM, CDR, MRP) did not. There was not a prior plan to evaluate the outcomes of AREDS2 treatment at the time supplementation was initiated. Patients with IMT2 were routinely followed every six to twelve months, although individual follow-up was variable and reflected clinical practice. As a result, the completeness of follow-up at 12 and 24 months favored these as time points for comparative analysis. At each encounter, best correct visual acuity (BCVA) and SDOCT images were obtained at a minimum in addition to other indicated testing. SD-OCT data analyzed included central macular thickness (CMT), length of ellipsoid zone (EZ) loss, and cystic cavitary diameter. AREDS2 eyes were eligible for inclusion if complete baseline data was available and there was at least one subsequent follow-up at 12 or 24 months after supplementation. Exclusion criteria were non-adherence to AREDS2 supplementation, AREDS2 treatment for non-exudative age-related macular degeneration, proliferative IMT2, endstage IMT2, severe intraretinal pigment migration, cataract greater than grade 2, cataract surgery during the study period, macular 
surgery and other visually significant pathology including choroidal neovascular membrane, macular scarring, diabetic macular edema, or visually significant epiretinal membrane. Furthermore, cases with diagnostic uncertainly or findings inconsistent with IMT2 were excluded.

The analysis was completed in two study arms. The non-comparative arm of the study involved all AREDS2 eyes meeting inclusion criteria. This portion of the study did not include a natural history control group. Given the inherent asymmetry between eyes of patients with IMT2, ${ }^{18}$ both eyes were included in the non-comparative arm if each met criteria for inclusion.

The objective of the comparative arm was to differentiate outcomes of AREDS2 treated eyes and those of a natural history control cohort. Given the inconsistent follow-up that often accompanies a retrospective design, this study arm only included eyes with complete baseline, 12 and 24 month data in an effort to limit bias. Therefore, AREDS2 eyes from the non-comparative study arm were further screened and eyes with incomplete data were excluded from the comparative AREDS2 cohort. The control cohort consisted of IMT2 eyes with complete data that were not started on AREDS2. The same exclusion criteria previously mentioned for AREDS2 were applied to untreated eyes during the review period. Baseline characteristics for untreated eyes were determined by the earliest clinical encounter within the 5 -year review period while baseline data were collected at the time of treatment initiation for AREDS2 eyes. Similar to the non-comparative arm, we included both eyes if each eye met inclusion, although best and worst eye analyses were performed to validate these findings.

The primary outcomes of this study were BCVA and SD-OCT structural changes including EZ loss length, greatest cavitary diameter, and CMT after 24 months of AREDS2 supplementation. Secondary outcomes included comparison of AREDS2 and untreated eyes for BCVA, CMT, largest cavitary diameter and EZ loss at 24 months. Data on all outcome measures were recorded at baseline, 12 and 24 months.

\section{Optical Coherence Tomography Assessment}

SD-OCT (Spectralis, ${ }^{\circledR} \quad$ Heidelberg Engineering, Heidelberg, Germany) images at each time interval were graded by two unmasked graders (TAB, MWM). The SDOCT protocol used for clinical practice at our institution was $513 \times 496$ scans at high speed with a 30-degree lens producing nineteen $5.7 \mathrm{~mm}$ raster scans. CMT measurements were completed with the thickness map function on the embedded automated software from the SD-OCT unit and were confirmed accurate by the grader. Measurements of cavitary diameters at baseline and follow-up encounters were completed with the caliper function of the embedded software, measuring the longest horizontal diameter. Similarly, EZ defect measurements were completed with the embedded caliper function after reviewing SD-OCT raster scans to locate an image with largest length of deficit. The same registered raster scan was compared on subsequent images across time for each anatomical characteristic.

\section{Statistical Analysis}

Snellen visual acuity measurements were converted to $\operatorname{logMAR}$ for analysis. However, Snellen acuities and letters are provided when appropriate. All means are represented as mean \pm standard deviation. In both study arms, mean difference values for each outcome were calculated respective to baseline as follows: baseline value minus follow-up value. A last value carried forward analysis was used to address missing follow-up SD-OCT and visual acuity data for AREDS2 eyes in the non-comparative arm. For this portion of the study, one-way analysis of variance (ANOVA) with repeated measurements were used to compare AREDS2 visual and anatomical outcomes over time. Statistical comparison of AREDS2 and untreated cohorts was performed with two-way ANOVA with repeated measurements. For best/worst BCVA analysis, if only one eye met inclusion criteria, the eye was automatically designated as the worse eye. Unpaired two-tailed Student's $t$-test and chi-squared analysis were used to calculate significance for baseline and demographical data. A $p$-value of $<0.05$ was considered statistically significant.

\section{Results}

A total of 320 subjects were screened over the 5-year study period of which 301 patients had a diagnosis of IMT2 based on compatible clinical features. Of IMT2 cases reviewed, 54 patients were started on AREDS2 with 43 patients ( 82 eyes) meeting criteria for inclusion [Figure 1]. Two patients (4 eyes) were intolerant to AREDS2 supplementation due to gastrointestinal side effects and four patients (7 eyes) reported non-adherence and therefore were removed from analysis. Twenty-seven AREDS2 eyes had complete data and were included in the 


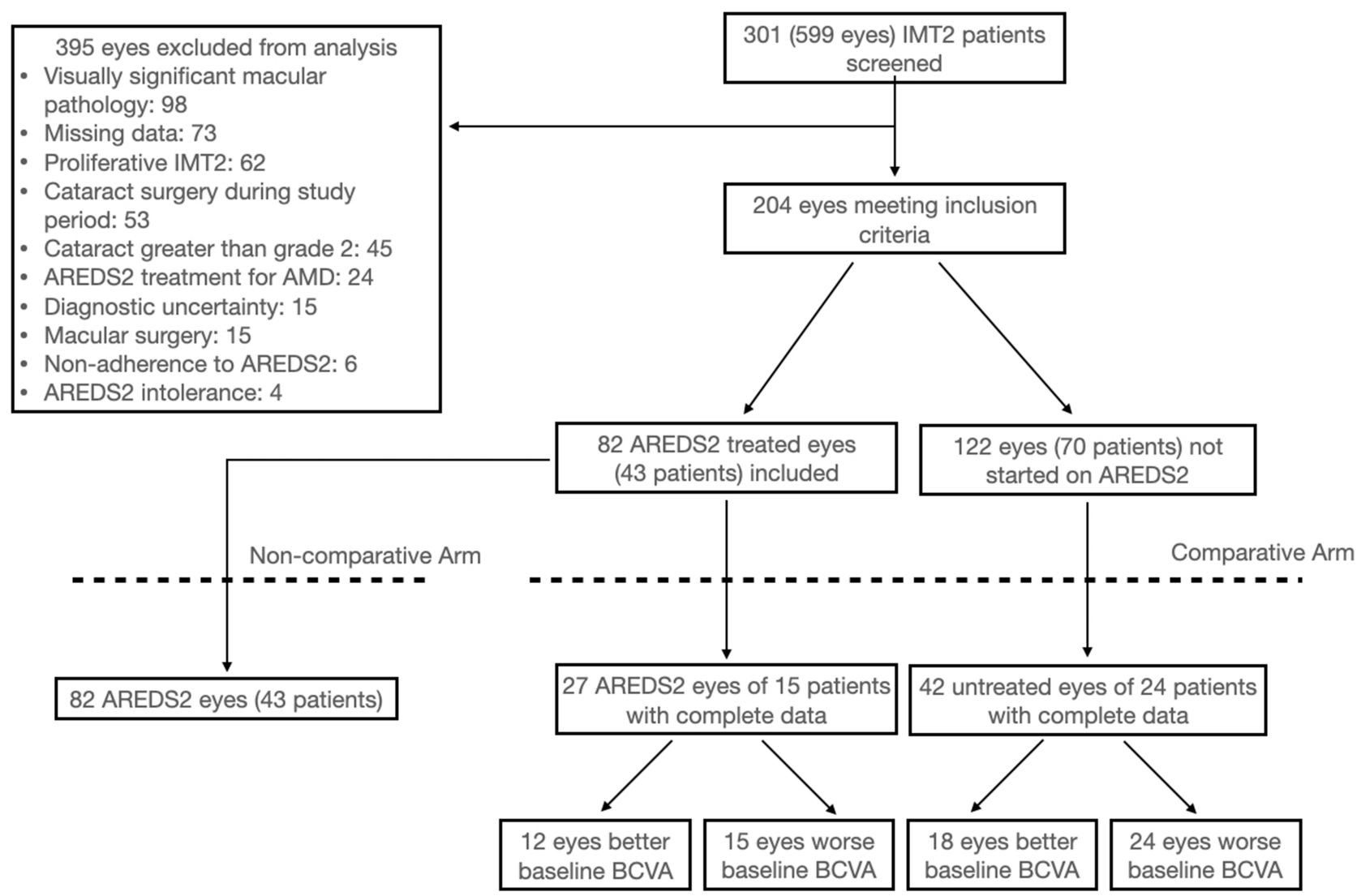

Figure I Study schematic demonstrating study design and cohort composition.

Abbreviations: AREDS2, Age-Related Eye Disease Study 2; AMD, age-related macular degeneration; IMT2, Idiopathic Macular Telangiectasia Type 2.

comparative aspect of the study. Of the 247 IMT2 patients not started on AREDS2, 24 patients (42 eyes) had complete data and also met inclusion criteria. These eyes were allocated to a natural history cohort. Demographical and baseline data for AREDS2 eyes included in the non-comparative arm are presented in Table 1. For the comparative arm, the AREDS2 and natural history cohorts had similar baseline BCVA and SD-OCT characteristics [Table 2].

\section{Visual Acuity}

For AREDS2 eyes in the non-comparative analysis, BCVA at 12 and 24 months were similar to baseline [Table 3]. Fifty-six (68.3\%) AREDS2 eyes had stable or improved visual acuity at 24 months. Comparatively, untreated eyes showed a progressive decline in BCVA at $12(-0.05 \pm 0.09$ $\log$ MAR, $-3.0 \pm 5.5$ letters vs $0.03 \pm 0.11 \log$ MAR, $1.8 \pm$ 6.6 letters) and 24 months $(-0.09 \pm 0.15,-5.4 \pm 8.5$ letters vs $0.03 \pm 0.11 \log$ MAR, $1.8 \pm 6.6$ letters) which was found to be significantly greater than eyes treated with ARED2 ( $\mathrm{p}=<0.001)$ [Table 4]. The net mean change in BCVA between the two cohorts was $0.12 \log$ MAR (7.2 letters) at the 24-month study endpoint. Better and worst eye analysis of BCVA was performed at 12 and 24 months. In both better and worse seeing eyes, untreated eyes had a

Table I Demographics and Baseline Characteristics for AREDS2 in the Non-Comparative Study Arm

\begin{tabular}{|l|l|}
\hline Eyes, no. & 82 \\
\hline Patients, no. & 43 \\
\hline Gender, male:female & $15: 28$ \\
\hline Laterality, right:left & $20: 21$ \\
\hline Age, years mean \pm SD & $64.3 \pm 10.8$ \\
\hline BCVA & \\
logMAR mean \pm SD & $0.28 \pm 0.18$ \\
Snellen & $20 / 38$ \\
\hline OCT data, mm mean \pm SD & \\
Central macular thickness & $246.8 \pm 27.4$ \\
EZ loss length & $500.5 \pm 529.8$ \\
Greatest cavitary diameter & $232.5 \pm 220.8$ \\
\hline
\end{tabular}

Abbreviations: ARED2, Age-Related Eye Disease Study 2; no., number; SD, standard deviation; BCVA, best corrected visual acuity; logMAR, logarithm of the minimum angle of resolution; OCT, optical coherence tomography; EZ, ellipsoid zone. 
Table 2 Demographics and Baseline Characteristics for Comparative Analysis of AREDS2 and Untreated Eyes

\begin{tabular}{|l|l|l|l|}
\hline & AREDS2 & Control & p value* \\
\hline Eyes, no. & 27 & 42 & \\
\hline Patients, no. & 15 & 24 & \\
\hline Gender, male:female & $7: 8$ & $7: 17$ & $22: 20$ \\
\hline Laterality, right:left & $13: 14$ & $66.3 \pm 7.6$ & 0.78 \\
\hline Age, years, mean \pm SD & $67.0 \pm 8.1$ & $2: 5$ & 0.45 \\
\hline $\begin{array}{l}\text { Lenticular status, } \\
\text { phakic:pseudophakic }\end{array}$ & $3: 5$ & $0.23 \pm 22.0$ & 0.72 \\
\hline $\begin{array}{l}\text { BCVA } \\
\text { logMAR mean } \pm \text { SD } \\
\text { Snellen }\end{array}$ & $0.25 \pm 17.2$ & $20 / 34$ & 0.54 \\
\hline $\begin{array}{l}\text { OCT data, mm, mean } \\
\pm \text { SD } \\
\text { Central macular } \\
\text { thickness } \\
\text { EZ loss length } \\
\begin{array}{l}\text { Greatest cavitary } \\
\text { diameter }\end{array}\end{array}$ & $\begin{array}{l}251.4 \pm 30.0 \\
238.2 \pm 251.5\end{array}$ & $233.4 \pm 208.4$ & 0.94 \\
\hline
\end{tabular}

Notes: ${ }^{*} p$ value reflects statistical comparison between AREDS2 and untreated eyes with unpaired t-tests for continuous data and chi square analysis for categorical values; $p<0.05$ denotes statistical significance.

Abbreviations: ARED2, Age-Related Eye Disease Study 2; no., number; SD, standard deviation; $B C V A$, best corrected visual acuity; logMAR, logarithm of the minimum angle of resolution; OCT, optical coherence tomography; EZ, ellipsoid zone.

significantly greater rate of visual decline compared to AREDS2 eyes after 24 months of supplementation ( $p=$ 0.01 and $\mathrm{p}=0.01$, respectively) [Table 5].

\section{SD-OCT Outcomes}

Representative SD-OCT examples of AREDS2 and untreated eyes are presented in Figure 2. In the noncomparative arm, EZ loss was similar to baseline for AREDS2 treated eyes after 24 months of supplementation $(p=0.98)$. The mean difference for EZ loss in the non-comparative arm was $-18.40 \pm 144.60 \mathrm{~mm}$ at 24 months. AREDS2 eyes showed reduced progression of EZ loss compared to untreated eyes during the study period $(p=0.02)$. After grouping by best/worse eye, untreated eyes had increased EZ loss over 24 months in worse seeing eyes $(p=0.04)$, although the rate of EZ loss was found to be similar for both cohorts in better seeing eyes $(\mathrm{p}=0.35)$.

Qualitative grading of SD-OCT images revealed macular cavitations in $65(79.3 \%)$ AREDS2 treated eyes at baseline. In the non-comparative arm, greatest cavitary
Table 3 Non-Comparative Study Arm Visual and OCT Outcomes for AREDS2 Eyes After 24 Months of Supplementation

\begin{tabular}{|l|l|l|l|}
\hline Interval, Month & $\mathbf{1 2}$ & $\mathbf{2 4}$ & *p value \\
\hline $\begin{array}{c}\text { BCVA, logMAR } \\
\text { Mean } \pm \text { SD }\end{array}$ & $0.27 \pm 0.18$ & $0.26 \pm 0.19$ & 0.86 \\
Mean difference \pm SD & $0.01 \pm 0.13$ & $0.02 \pm 0.13$ & \\
\hline $\begin{array}{c}\text { EZ loss length, mm } \\
\text { Mean } \pm \text { SD }\end{array}$ & $492.7 \pm 496.5$ & $514.2 \pm 499.8$ & 0.9 \\
Mean difference \pm SD & $3.15 \pm 120.11$ & $-18.40 \pm 144.60$ & \\
\hline $\begin{array}{c}\text { GCD, mm } \\
\text { Mean } \pm \text { SD }\end{array}$ & $219.7 \pm 240.6$ & $231.3 \pm 245.7$ & 0.98 \\
Mean difference $\pm S D$ & $12.82 \pm 97.80$ & $1.18 \pm 95.39$ & \\
\hline CMT, mm & & & \\
Mean \pm SD & $245.7 \pm 28.8$ & $247.8 \pm 28.4$ & 0.94 \\
Mean difference \pm SD & $1.05 \pm 12.52$ & $-2.86 \pm 17.05$ & \\
\hline Total, number (percent) & $54(60)$ & $44(54)$ & \\
\hline
\end{tabular}

Notes: * $p$ value reflects outcome of one-way analysis of variance (ANOVA) with repeated measurements. $p<0.05$ is considered statistically significant.

Abbreviations: OCT, optical coherence tomography; ARED2, Age-Related Eye Disease Study 2; $\log M A R$, logarithm of the minimum angle of resolution; SD, standard deviation; BCVA, best corrected visual acuity; EZ, ellipsoid zone; GCD, greatest cavitary diameter; CMT, central macular thickness.

diameters at 12 and 24 months were similar to baseline for AREDS2 eyes. Compared to AREDS2 eyes, untreated eyes showed a significant increase in cavitary diameter over 24 months $(\mathrm{p}=0.01)$. Mean difference in cavity diameter in better seeing eyes was similar for AREDS2 and untreated eyes $(p=0.24)$. In contrast, eyes with worse baseline BCVA demonstrated greater mean cavitary diameter in untreated eyes compared to AREDS2 eyes ( $p=$ $0.001)$.

Central macular thickness remained stable for AREDS2 treated eyes throughout the study period. In the comparative arm, CMT remained relatively stable for both cohorts and comparison of CMT mean difference over time was not significant.

\section{Discussion}

We investigated the use of off-label AREDS2 for the treatment of non-proliferative IMT2. We believe this is the first study investigating carotenoid supplementation in IMT2 which includes an untreated control group. Our results suggest that AREDS2 may slow anatomical and visual deterioration in non-proliferative IMT2. For BCVA, AREDS2 eyes remained stable throughout the study period with a mean difference from baseline of $0.02 \pm 0.13$ 
Table 4 AREDS2 vs Untreated Eyes BCVA and Optical Coherence Tomography Outcomes

\begin{tabular}{|c|c|c|c|c|c|c|}
\hline \multicolumn{7}{|c|}{ Month } \\
\hline & \multirow{2}{*}{$\begin{array}{l}\text { Baseline } \\
\text { Mean }\end{array}$} & \multicolumn{2}{|l|}{12} & \multicolumn{3}{|l|}{24} \\
\hline & & Mean & Mean Difference & Mean & Mean Difference & $p$ value* \\
\hline \multicolumn{7}{|c|}{ BCVA, logMAR } \\
\hline AREDS & $0.25 \pm 0.17$ & $0.25 \pm 0.18$ & $0.03 \pm 0.11$ & $0.22 \pm 0.19$ & $0.03 \pm 0.11$ & $<0.001$ \\
\hline Untreated & $0.23 \pm 0.22$ & $0.28 \pm 0.22$ & $-0.05 \pm 0.09$ & $0.32 \pm 0.23$ & $-0.09 \pm 0.15$ & \\
\hline \multicolumn{7}{|c|}{$\mathrm{EZ}$ loss length, $\mathrm{mm}$} \\
\hline AREDS & $630.7 \pm 532.7$ & $588.8 \pm 493.9$ & $41.9 \pm 141.2$ & $642.2 \pm 481.7$ & $-11.5 \pm 131.9$ & 0.02 \\
\hline Untreated & $548.7 \pm 489.7$ & $585.5 \pm 508.0$ & $-36.8 \pm 108.7$ & $617.7 \pm 522.2$ & $-69.0 \pm 137.6$ & \\
\hline \multicolumn{7}{|l|}{$\mathrm{GCD}, \mathrm{mm}$} \\
\hline AREDS & $238.2 \pm 251.2$ & $191.2 \pm 257.2$ & $47.0 \pm 78.0$ & $196.3 \pm 248.1$ & $41.9 \pm 109.3$ & 0.01 \\
\hline Untreated & $233.5 \pm 208.4$ & $254.0 \pm 226.8$ & $-20.6 \pm 90.3$ & $254.8 \pm 219.3$ & $-21.1 \pm 94.1$ & \\
\hline \multicolumn{7}{|l|}{ CMT, mm } \\
\hline AREDS & $251.4 \pm 30.0$ & $251.7 \pm 29.5$ & $-0.4 \pm 9.13$ & $255.2 \pm 32.4$ & $-3.8 \pm 20.7$ & 0.36 \\
\hline Untreated & $246.5 \pm 33.2$ & $244.1 \pm 31.9$ & $2.4 \pm 8.5$ & $246.0 \pm 38.8$ & $0.4 \pm 20.6$ & \\
\hline
\end{tabular}

Notes: All data presented as mean or mean difference \pm standard deviation; *p value reflects outcome for two-way analysis of variance (ANOVA) with repeated measurements for AREDS2 vs untreated eyes; $p<0.05$ is considered statistically significant.

Abbreviations: ARED2, Age-Related Eye Disease Study 2; BCVA, best corrected visual acuity; logMAR, logarithm of the minimum angle of resolution; EZ, ellipsoid zone; GCD, greatest cavitary diameter; CMT, central macular thickness.

$\log$ MAR $(1.2 \pm 7.8$ letters $)$ at 24 months. It is well established that IMT2 is a slowly progressive neurodegenerative disease often with decreased subjective visual quality preceding clinically evident visual acuity decline. ${ }^{9}$ The MacTel Project demonstrated a 1.1 letter per year visual deterioration which is less than the approximately 5 letter decline observed in our natural history cohort at 24 months. ${ }^{19}$ The reason for this is unclear but may be related to reduced statistical power and wide standard deviations observed in the comparative arm of the study. Nonetheless, the 5 letter decline over the 24-month study period is comparable to a natural history study of similar statistical power to the present study. ${ }^{20}$ The greater than expected visual decline of the control group may limit the comparative outcomes of this study; however, the non-comparative BCVA results at minimum suggest AREDS2 supplementation preserves visual acuity.

Treatment of non-proliferative IMT2 with AREDS2 is associated with anatomical stability on SD-OCT which may represent the basis for improved visual outcome. After 24 months of supplementation, EZ loss remained stable in the non-comparative arm and AREDS2 treated eyes showed less EZ loss progression compared to the untreated natural history cohort. Several groups have demonstrated that EZ loss is predictive of visual acuity loss in IMT2 $2^{17,20-22}$ and particularly EZ loss involving the foveal center. ${ }^{19,21}$ An important consideration when evaluating this study's EZ outcomes is the use of length to quantify EZ defects. EZ loss is typically quantified by area via en face OCT analysis which was not available within the scan parameters routinely used in clinical practice at the time of the study. Despite this limitation, Pauleikhoff et al found linear EZ break length to be a reliable surrogate for EZ area loss less than $0.15 \mathrm{~mm}^{2}$ in IMT2 eyes. ${ }^{21}$ The rate of EZ loss observed in the present study's natural history cohort is consistent with prior natural history studies in the literature. ${ }^{7,20,22}$ Assuming EZ loss is no greater than a circular area and the EZ length observed on SDOCT represents the longest dimension, EZ loss length can be approximated to EZ area as follows:

$$
\begin{aligned}
& \begin{array}{l}
\text { Approximate EZ } \\
\text { change in area }
\end{array}=\frac{\frac{\text { (baseline EZ length-2 year EZ length) }}{2} \times \pi}{1000} \\
& \text { AREDS } 2 \text { cohort }=\frac{5.75 \times \pi}{1000}=0.02 \mathrm{~mm}^{2} \\
& \text { Untreated cohort }=\frac{34.5 \mathrm{x} \pi}{1000}=0.11 \mathrm{~mm}^{2}
\end{aligned}
$$

Therefore, the rate of EZ loss observed in our untreated cohort is comparable to previously reported natural history data from the Mactel Project and ciliary neurotrophic factor clinical trial and provides validity for the rate of EZ loss observed in the natural history cohort. ${ }^{7,19}$ 
Table 5 OCT and Visual Outcomes After Better/Worse Baseline Visual Acuity Analysis for AREDS vs Untreated Eyes

\begin{tabular}{|c|c|c|c|c|c|c|}
\hline \multirow[t]{2}{*}{ Interval, Month } & \multirow{2}{*}{$\begin{array}{l}\text { Baseline } \\
\text { Mean }\end{array}$} & \multicolumn{2}{|l|}{12} & \multicolumn{2}{|l|}{24} & \multirow[t]{2}{*}{ *p value } \\
\hline & & Mean & Mean Difference & Mean & Mean Difference & \\
\hline \multicolumn{7}{|c|}{ Better BCVA eyes (AREDS2 $n=12$; Untreated $n=18$ ) } \\
\hline \multicolumn{7}{|l|}{ BCVA, logMAR } \\
\hline AREDS2 & $0.15 \pm 0.09$ & $0.19 \pm 0.17$ & $-0.03 \pm 0.13$ & $0.11 \pm 0.08$ & $0.04 \pm 0.09$ & 0.01 \\
\hline Untreated & $0.18 \pm 0.17$ & $0.23 \pm 0.20$ & $-0.05 \pm 0.10$ & $0.24 \pm 0.21$ & $-0.07 \pm 0.15$ & \\
\hline \multicolumn{7}{|l|}{ EZ loss length, mm } \\
\hline AREDS2 & $448.7 \pm 479.4$ & $450.7 \pm 471.0$ & $-2.0 \pm 95.3$ & $472.5 \pm 456.4$ & $-23.8 \pm 152.2$ & 0.35 \\
\hline Untreated & $394.5 \pm 421.9$ & $436.1 \pm 473.8$ & $-41.6 \pm 129.1$ & $456.8 \pm 466.7$ & $-62.4 \pm|4| .2$ & \\
\hline \multicolumn{7}{|l|}{$\mathrm{GCD}, \mathrm{mm}$} \\
\hline AREDS2 & $144.7 \pm 163.6$ & $126.7 \pm 164.8$ & $18.0 \pm 34.9$ & $131.0 \pm 155.1$ & $13.7 \pm 73.9$ & 0.34 \\
\hline Untreated & $297.3 \pm 169.4$ & $335.4 \pm 205.2$ & $-38.2 \pm 98.1$ & $305.3 \pm 181.5$ & $-8.1 \pm 126.2$ & \\
\hline \multicolumn{7}{|l|}{ CMT, mm } \\
\hline AREDS2 & $246.5 \pm 24.8$ & $249.8 \pm 23.8$ & $-3.1 \pm 10.4$ & $255.8 \pm 24.8$ & $-9.0 \pm 24.8$ & 0.17 \\
\hline Untreated & $249.0 \pm 33.2$ & $246.3 \pm 32.7$ & $1.9 \pm 6.1$ & $249.9 \pm 41.8$ & $2.1 \pm 16.7$ & \\
\hline \multicolumn{7}{|c|}{ Worse BCVA (AREDS2 $n=15 ;$ Untreated $n=24$ ) } \\
\hline \multicolumn{7}{|l|}{ BCVA, logMAR } \\
\hline AREDS2 & $0.34 \pm 0.17$ & $0.31 \pm 0.17$ & $0.03 \pm 0.11$ & $0.34 \pm 0.34$ & $0.01 \pm 0.12$ & 0.01 \\
\hline Untreated & $0.27 \pm 0.25$ & $0.31 \pm 0.24$ & $-0.04 \pm 0.09$ & $0.38 \pm 0.15$ & $-0.11 \pm 0.24$ & \\
\hline \multicolumn{7}{|l|}{ EZ loss length, mm } \\
\hline AREDS2 & $776.3 \pm 524.7$ & $699.3 \pm 483.8$ & $77.0 \pm 164.1$ & $777.9 \pm 472.1$ & $-1.7 \pm 117.9$ & 0.04 \\
\hline Untreated & $648.0 \pm 513.2$ & $679.4 \pm 512.0$ & $-32.8 \pm 91.4$ & $750.7 \pm 537.8$ & $-74.5 \pm 137.6$ & \\
\hline \multicolumn{7}{|l|}{$\mathrm{GCD}, \mathrm{mm}$} \\
\hline AREDS2 & $311 \pm 280.8$ & $247.6 \pm 300.3$ & $63.4 \pm 98.1$ & $248.1 \pm 290.6$ & $66.6 \pm 126.2$ & 0.001 \\
\hline Untreated & $185.6 \pm 225.0$ & $192.9 \pm 226.9$ & $-68.7 \pm 62.4$ & $216.9 \pm 240.5$ & $-3 \mid .3 \pm 85.0$ & \\
\hline \multicolumn{7}{|l|}{ CMT, mm } \\
\hline AREDS2 & $254.6 \pm 32.2$ & $252.6 \pm 32.3$ & $2.1 \pm 7.0$ & $254.7 \pm 36.4$ & $0.2 \pm 16.7$ & 0.94 \\
\hline Untreated & $249.0 \pm 33.2$ & $246.3 \pm 33.2$ & $2.7 \pm 10.2$ & $249.9 \pm 41.8$ & $-0.9 \pm 23.6$ & \\
\hline
\end{tabular}

Notes: All data are presented as mean or mean difference \pm standard deviation; *p value reflects outcome of two-way analysis of variance (ANOVA) with repeated measurements for AREDS2 vs untreated eyes, $p<0.05$ is considered statistically significant.

Abbreviations: ARED2, Age-Related Eye Disease Study 2; OCT, optical coherence topography; BCVA, best corrected visual acuity; logMAR, logarithm of the minimum angle of resolution; EZ, ellipsoid zone; GCD, greatest cavitary diameter; CMT, central macular thickness mes after better/worse baseline visual acuity analysis for AREDS vs untreated eyes.

A hallmark of SD-OCT findings in IMT2 are the formation of hyporeflective intra-retinal cavities. ${ }^{9,23}$ Previously, Tan et al reported a trend towards reduced greatest cavity diameter after 6 months of supplementation with lutein, meso-zeaxanthin and zeaxanthin. Our results showed stability of greatest cavitary diameter after 24 months of AREDS2 supplementation. We recognize that the intra-retinal cysts may be transient and fluctuate over time. ${ }^{9}$ While EZ loss is likely a better predictor of visual function, a recent study by Cai et al demonstrated an association between worse BCVA and cavitary size and expansion. ${ }^{23}$ Therefore, it is possible the stability of cavitary size observed in AREDS2 eyes may indicate decreased neurodegeneration and therefore may additionally contribute to an improved visual outcome.

CMT remained stable for AREDS2 eyes throughout the study period and comparatively was similar to the natural history cohort at 24 months. This result is similar to CMT outcomes of prior studies examining carotenoid supplementation in IMT2. ${ }^{14,15}$

Interestingly, in eyes with worse baseline visual acuity, AREDS2 showed a reduction in greatest cavitary diameter and EZ loss length compared to untreated eyes after 24 months of supplementation while no significant difference was appreciated in eyes with better baseline BCVA. Based on prior observations that areas with macular 


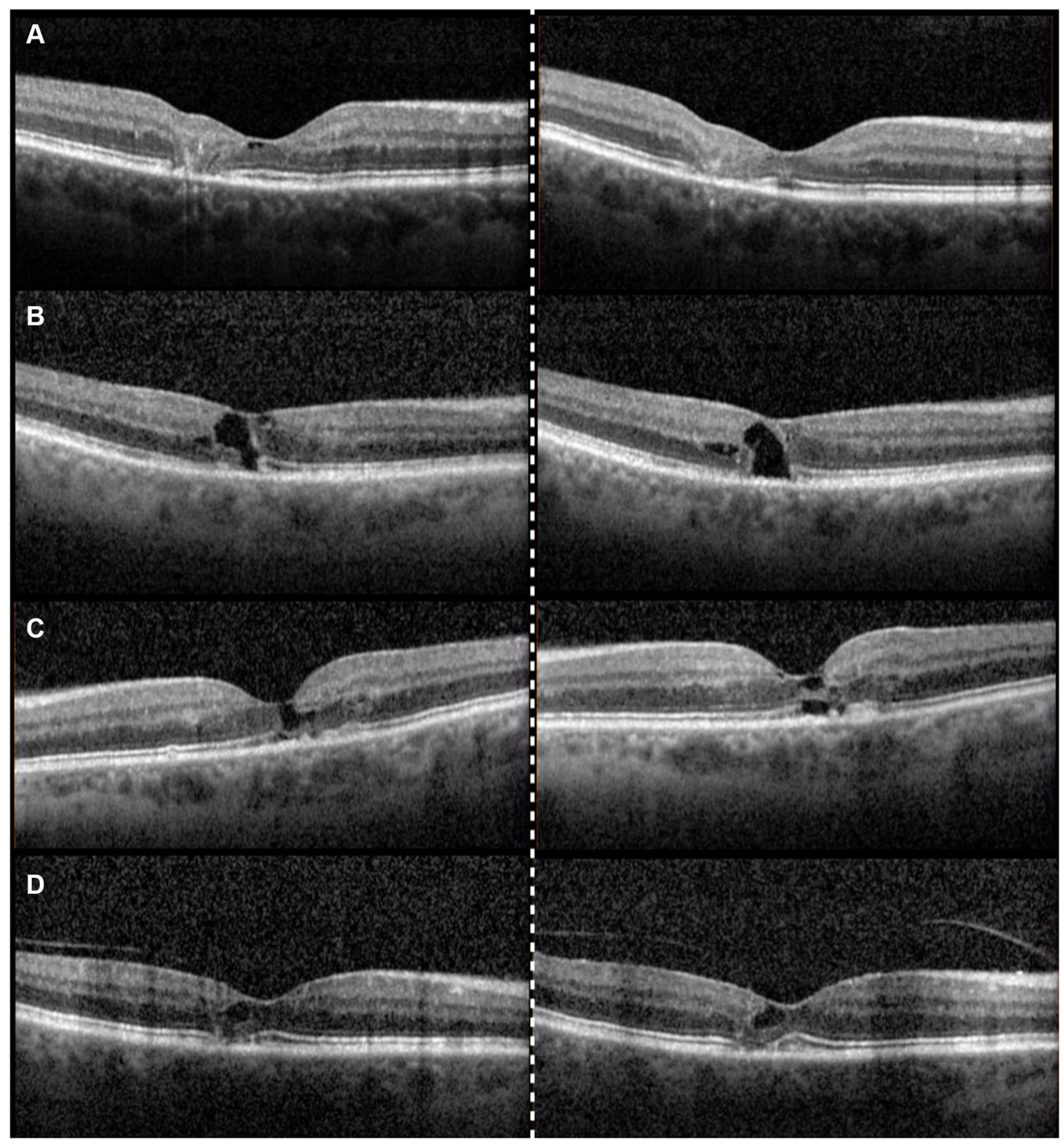

Figure 2 Representative examples of ellipsoid zone (EZ) loss on spectral domain optical coherence tomography (SD-OCT) for untreated (A-B) and Age-Related Eye Disease Study 2 (AREDS2) treated eyes (C-D). SD-OCT images on the right show baseline imaging while the left is from 2-year follow-up. (A) showing an untreated eye at baseline with $20 / 30^{-1}$ visual acuity and an EZ defect of $488 \mathrm{~mm}$. Subsequent 2 years follow-up reveals a worsening EZ defect of $617 \mathrm{~mm}$ and stable VA of $20 / 30^{-1}$. (B) depicts an untreated eye with $20 / 30^{-2}$ vision and $262 \mathrm{~mm}$ of EZ loss. At 2 years, vision acuity declines to $20 / 40^{+2}$ and EZ loss progressed to $568 \mathrm{~mm}$. (C) is of a AREDS2 treated eye with baseline visual acuity of $20 / 25^{+2}$ and EZ defect of 467 , both of which remain relatively similar at 2 years (20/20 and $\left.474 \mathrm{~mm}\right)$. (D) showing an AREDS2 treated eye with $20 / 30^{-1}$ baseline visual acuity and $272 \mathrm{~mm}$ EZ loss length. Visual acuity and EZ loss length remain stable at 2 years at 20/30 and $285 \mathrm{~mm}$, respectively.

depigmentation do not appear to re-establish pigment with carotenoid supplementation, ${ }^{14,15}$ one might expect earlier disease to be more responsive to AREDS2. The lack of significance for EZ loss and cavitary diameter may be explained by loss of statistical power after further division of eyes for better/worse eye analysis. Alternatively, this may indeed suggest eyes with moderate disease receive a more pronounced benefit from supplementation. EZ loss in 
IMT2 is non-linear and follows an exponential increase before reaching a plateau. ${ }^{20}$ Likewise, visual acuity follows a similar trend as it may remain relatively preserved until later disease when EZ loss involves the fovea. ${ }^{19,21}$ These non-linear relationships suggest AREDS2's stabilizing effect may exert a greater magnitude in symptomatic patients than earlier in the disease process. However, we specifically excluded endstage eyes, which would not be expected to benefit from a stabilizing therapy.

The results of the present study suggest that AREDS2 may represent a novel treatment for non-proliferative IMT2. Considerable attention has been drawn to macular pigment loss in IMT2, which has generated an interest in the use carotenoids for the IMT2 treatment. Lutein and zeaxanthin are thought to provide a beneficial physiological effect through the reduction of oxidative stress by serving as local free radical scavengers and filtering blue light. $^{24,25}$ However, it is important to acknowledge the potential role of the antioxidant effect of zinc, vitamin $\mathrm{C}$, and vitamin $\mathrm{E}$ in the outcome of this study. A beneficial effect of these antioxidants without lutein and zeaxanthin has been observed in non-exudative age-related macular degeneration in the AREDS study. ${ }^{26}$ Numerous studies have demonstrated Müller cell susceptibility to oxidative damage $^{27-30}$ and there is some evidence from mouse models that oxidative stress may contribute to the pathogenesis of IMT2. ${ }^{27}$

Our study had several limitations which must be taken in account when analyzing our results. First, our study design is limited by a retrospective design with imaging per standard clinic protocol rather than a study protocol with intent for prospective analysis. Additional limitations regarding the study design include unmasked graders and lack of intent-to-treat analysis. Selection bias may have influenced the composition of both cohorts, as the studied cohorts represent a small fraction of all the identified IMT2 patients. The control group in this study was not intentionally matched to reduce confounding bias. However, there was no statistical difference between baseline SD-OCT, BCVA and demographical data amongst AREDS2 and untreated eyes. Given the slowly progressive nature of this neurodegenerative disease, another limitation of this study was the selection of 24 months as the study endpoint. We reviewed the data available on AREDS2 eyes at 36 months and observed substantial decline in follow-up data limiting the utility of extending the study end point and determining a meaningful outcome. Future studies with a prospective design will be able to provide greater, more complete longitudinal data. Measurement of EZ loss by length rather than en face area was a limitation in our study. However, en face OCT analysis could not be performed based upon the scan parameters routinely used in clinical practice during the study period. A confirmatory randomized, prospective clinical trial should utilize en face imaging and microperimetry as a functional outcome given the slow change in visual acuity over time.

\section{Conclusion}

We conclude that off-label AREDS2 supplementation in non-proliferative IMT2 is associated with stabilization of structural deterioration and visual decline. This is the largest study investigating carotenoid and antioxidant supplementation for IMT2 and the first with a natural history cohort. While the comparative arm of this study is limited by statistical power, the stabilizing benefits of AREDS2 in this study were similar to the outcomes observed in the Phase 2 encapsulated cell technology trial. Given the minimal risk of severe adverse events, non-invasive delivery, and low cost of AREDS2, further exploration with a prospective, randomized clinical trial is warranted.

\section{Disclosure}

Dr Christopher D Riemann reports personal fees from Alcon, CVP (CEI Vision Partners), ForwardVue Pharma, Gore, Gyroscope, Haag Streit, NotalVision LLC, Novartis, Orbit BioMedical, Regeneron, Reliance Industries, Salutaris MD, Samsara, TrueVision, VEO Ophthalmics, speaker and consultant for Alimera, Allergan, Bausch \& Lomb/Valeant, BMC/Eyetube, consultant for Animal Eye Institute, D.O.R.C., HumanOptics AG, Lineage/BioTime, Medical and Scientific Advisory Council for Aniridia Foundation International, speaker for CSTLII, owner/ cofounder of Chruman Research, Board of Trustees for Clovernook Center for the Blind and Visually Impaired, stock and ownership of Digital Surgery Systems, consultant, cofounder, and IP for Iamc2, investor, stock, and consultant for iVeena, consultant and I.P. for Janssen/ Johnson \& Johnson, Kaleidoscope Engineering, consultant, royalties, I.P. for/from MedOne, personal fees from and ownership of Northmark Pharmacy, investor, stock, consultant, royalties, I.P. for/from Vortex Surgical, and monies for research from AGTC, Alcon, Alimera, Allergan, Arepio, BioTime/Lineage, Chengdu Kanghong, Clearside, Genentech/Roche, Gyroscope, Lineage/ BiotimeJanssen/Johnson \& Johnson, Lowry-MacTel 
Registry, Neurotech, Nightstar/Biogen, NotalVision, Novartis, Ophthotec/Iveric, Regeneron, and Spark, during the conduct of the study. Dr Robert A Sisk reports consulting for AGTC, Gyroscope, Allergan/Abbvie, RegenXBio, Leica, and EyePoint, outside the submitted work. The authors report no other potential conflicts of interest in this work.

\section{References}

1. Sigler EJ, Randolph JC, Calzada JI, et al. Comparison of observation, intravitreal bevacizumab, or pars plana vitrectomy for nonproliferative type 2 idiopathic macular telangiectasia. Graefe's Arch Clin Exp Ophthalmol. 2013;251(4):1097-1101. doi:10.1007/ s00417-012-2150-1

2. Charbel Issa P, Finger RP, Kruse K, et al. Monthly Ranibizumab for Nonproliferative Macular Telangiectasia Type 2: a 12-Month Prospective Study. Am J Ophthalmol. 2011;151(5):876-886. doi:10. 1016/j.ajo.2010.11.019

3. Wu L, Evans T, Arévalo JF, et al. Long-term effect of intravitreal triamcinolone in the nonproliferative stage of type II idiopathic parafoveal telangiectasia. Retina. 2008;28(2):314-319. doi:10.1097/ IAE.0b013e31814cf03e

4. De Lahitte GD, Cohen SY, Gaudric A. Lack of apparent short-term benefit of photodynamic therapy in bilateral, acquired, parafoveal telangiectasis without subretinal neovascularization. $\mathrm{Am} \mathrm{J}$ Ophthalmol. 2004;138(5):892-894. doi:10.1016/j.ajo.2004.06.010

5. Park DW, Schatz H, McDonald HR, Johnson RN. Grid laser photocoagulation for macular edema in bilateral juxtafoveal telangiectasis. Ophthalmology. 1997;104(11):1838-1846. doi:10.1016/S0161-6420 (97)30019-0

6. Chew EY, Clemons TE, Peto T, et al. Ciliary neurotrophic factor for macular telangiectasia type 2: results from a Phase 1 safety trial. $\mathrm{Am}$ J Ophthalmol. 2015;159(4):659-666. doi:10.1016/j.ajo.2014.12.013

7. Chew EY, Clemons TE, Jaffe G, et al. Effect of ciliary neurotrophic factor on retinal neurodegeneration in patients with macular telangiectasia Type 2. Ophthalmology. 2019;126(4):540-549. doi:10.1016/ j.ophtha.2018.09.041

8. Gass JD, Blodi BA. Idiopathic juxtafoveolar retinal telangiectasis. Update of classification and follow-up study. Ophthalmology. 1993;100(10):1536-1546. doi:10.1016/S0161-6420(93)31447-8

9. Charbel Issa P, Gillies MC, Chew EY, et al. Macular telangiectasia type 2. Prog Retin Eye Res. 2013;34:49-77. doi:10.1016/j.preteyeres. 2012.11.002

10. Gantner ML, Eade K, Wallace M, et al. Serine and lipid metabolism in macular disease and peripheral neuropathy. $N$ Engl J Med. 2019;381:1422. doi:10.1056/NEJMoa1815111

11. Zeimer MB, Padge B, Heimes B, et al. Idiopathic macular telangiectasia type 2: distribution of macular pigment and functional investigations. Retina. 2010;30(4):586-595. doi:10.1097/IAE.0b013e3181 bd2d 38

12. Powner MB, Gillies MC, Tretiach M, et al. Perifoveal müller cell depletion in a case of macular telangiectasia type 2. Ophthalmology. 2010;117(12):2407-2416. doi:10.1016/j.ophtha.2010.04.001

13. Powner MB, Gillies MC, Zhu M, et al. Loss of müllers cells and photoreceptors in macular telangiectasia type 2. Ophthalmology. 2013;120(11):2344-2352. doi:10.1016/j.ophtha.2013.04.013

14. Zeimer MB, Isabel I. Spital, G et al. Macular telangiectasia patterns of distribution of macular pigment and Response to Supplementation. Retina. 2010;30(8):1282-1293. doi:10.1097/IAE.0b013e3181e096dd
15. Choi RY, Gorusupudi A, Wegner K, et al. Macular pigment distribution responses to high-dose zeaxanthin supplementation in patients with macular telangiectasia type 2. Retina. 2017;37(12):2238-2247. doi:10.1097/IAE.0000000000001450

16. Tan ACS, Balaratnasingam C, Yannuzzi LA. Treatment of macular telangiectasia type 2 with carotenoid supplements containing mesozeaxanthin: a pilot study. Ophthalmic Surg Lasers Imaging Retina. 2016;47(6):528-535. doi:10.3928/23258160-20160601-04

17. Yannuzzi LA, Bardal AMC, Freund KB, et al. Idiopathic Macular Telangiectasia. Arch Ophthalmol. 2006;124(4):450-460. doi:10.1001/ archopht.124.4.450

18. Heeren TFC, Chew EY, Clemons T, et al. macular telangiectasia type 2: visual acuity, disease end stage, and the macTel area: macTel project report number 8. Ophthalmology. 2020;127(11):1539-1548. doi:10.1016/j.ophtha.2020.03.040

19. Peto T, Heeren TFC, Clemons TE, et al.Correlation of clinical and structural progression with visual acuity loss in macular telangiectasia type 2. Retina. 2018;(38):8-13. doi:10.1097/IAE.000000000 0001697

20. Heeren TFC, Kitka D, Florea D, et al.Longitudinal correlation of ellipsoid zone loss and functional loss in macular telangiectasia type 2. Retina. 2018;(38):20-26. doi:10.1097/IAE.0000000000001715

21. Pauleikhoff D, Bonelli R, Dubis AM, et al. Progression characteristics of ellipsoid zone loss in macular telangiectasia type 2. Acta Ophthalmol. 2019;97:998-1005. doi:10.1111/aos.14110

22. Sallo FB, Peto T, Egan C, et al. "En face" OCT imaging of the IS/OS junction line in type 2 idiopathic macular telangiectasia. Invest Ophthalmol Vis Sci. 2012;53(10):6145-6152. doi:10.1167/iovs.1210580

23. Cai CX, Choong J, Farsiu S, et al. Retinal cavitations in macular telangiectasia type 2 (MacTel): longitudinal structure-function correlations. $\mathrm{Br} J$ Ophthalmol. 2021;105(1):109-112. doi:10.1136/ bjophthalmol-2019-315416

24. Lima VC, Rosen RB, Farah M. Macular pigment in retinal health and disease. Int J Retina Vitreous. 2016;2:1. doi:10.1186/s40942-0160044-9

25. Whitehead AJ, Mares JA, Danis RP. Macular pigment: a review of current knowledge. Arch Ophthalmol. 2006;124(7):1038-1045. doi:10.1001/archopht.124.7.1038

26. Age-Related Eye Disease Study Research Group. A randomized, placebo-controlled, clinical trial of high-dose supplementation with vitamins $\mathrm{C}$ and $\mathrm{E}$, beta carotene, and zinc for age-related macular degeneration and vision loss: AREDS report no. 8. Arch Ophthalmol. 2001;119(10):1417-1436. doi:10.1001/archopht.119.10.1417

27. Dorrell MI, Aguilar E, Jacobson R, et al. Antioxidant or neurotrophic factor treatment preserves function in a mouse model of neovascularization-associated oxidative stress. J Clin Invest. 2009;119(3):611623. doi:10.1172/JCI35977

28. Cheng Z, Zhang T, Zheng J, et al. Betulinic acid derivatives can protect human Müller cells from glutamate-induced oxidative stress. Exp Cell Res. 2019;383:1. doi:10.1016/j.yexcr.2019.111509

29. Toft-Kehler AK, Gurubaran IS, Desler C, et al. Oxidative stressinduced dysfunction of müller cells during starvation. Invest Ophthalmol Visual Sci. 2016;57(6):2721-2728. doi:10.1167/iovs.1619275

30. Zhang X, Feng Z, Li C, Zheng Y. Morphological and migratory alterations in retinal müller cells during early stages of hypoxia and oxidative stress. Neural Regen Res. 2012;7(1):31-35. doi:10.3969/j. issn.1673-5374.2012.01.005 


\section{Publish your work in this journal}

Clinical Ophthalmology is an international, peer-reviewed journal covering all subspecialties within ophthalmology. Key topics include: Optometry; Visual science; Pharmacology and drug therapy in eye diseases; Basic Sciences; Primary and Secondary eye care; Patient Safety and Quality of Care Improvements. This journal is indexed on PubMed

Submit your manuscript here: https://www.dovepress.com/clinical-ophthalmology-journal
Central and CAS, and is the official journal of The Society of Clinical Ophthalmology (SCO). The manuscript management system is completely online and includes a very quick and fair peer-review system, which is all easy to use. Visit http://www.dovepress.com/ testimonials.php to read real quotes from published authors. 